\title{
THE EXPANSION OF A FUNCTION IN TERMS OF
}

\section{NORMAL FUNCTIONS.*}

BY

\section{MAX MASON.}

In an article published in these Transactions $\dagger$ the author has proved the existence of normal functions for the differential equation

$$
y^{\prime \prime}+p(x) y^{\prime}+[\lambda A(x)-B(x)] y=0
$$

and the boundary conditions

$$
\left\{\begin{array}{l}
a_{1} y(a)+a_{2} y(b)+a_{3} y^{\prime}(a)+a_{4} y^{\prime}(b)=0, \\
b_{1} y(a)+b_{2} y(b)+b_{3} y^{\prime}(a)+b_{4} y^{\prime}(b)=0,
\end{array}\right.
$$

by a method which sets in evidence the properties of the normal functions as solutions of minimum problems. It was shown that this point of view is useful in deriving oscillation theorems, i. e., in investigating the zeros of the functions.

It is the object of this paper to show that the minimal properties of the normal functions may be used to prove in a simple manner the possibility of expanding a function in terms of normal functions. $\ddagger$

The coefficients $p, A, B$ are supposed to be continuous in the interval $(a, b)$. In addition the following assumptions are made, the determinants $a_{i} b_{k}-a_{k} b_{i}$ of the coefficients of the equations (2) being denoted by $d_{i k}$ :

\footnotetext{
* Presented to the Society September 3 and December 28, 1906, in less general form. Received for publication June 27, 1907.

+ Vol. 7 (1906), p. 337.

$\ddagger$ Recent articles of special importance on this subject are those of HILBERT, Grundzüge einer allgemeinen Theorie der linearen Integralgleichungen, Nachrichten der K. Gesellschaft der Wissenschaften zu Göttingen, 1904, 1905, 1906, and KNESER, Mathematische Annalen, vol. 63 (1907), p. 477. These articles are based on the theory of integral equations, as developed by HILBERT and SchMidT. KNESER removes the restrictions imposed by HiLBERT, that the function be continuous with its first and second derivatives, and satisfy the same boundary conditions as the normal functions. In all work on this subject the boundary conditions for the normal functions are special cases of equations (2), above, except in the important case of singularities of the differential equation. The function $A$ is supposed positive in all previous work, except in the fifth memoir of HILBERT's series, in which, by considering a new class of integral equations, the restriction is removed. This restriction will not be made in the present article.
} 


$$
B(x) \geqq 0
$$$$
(a \leqq x \leqq b) \text {, }
$$

(y) No two of the quantities $d_{12}, d_{14}, d_{23}, d_{43}$ have opposite signs.

Equation $(\beta)$ insures that any two functions $u, v$ which satisfy the boundary conditions (2) also satisfy the equation *

$$
\left[P\left(u v^{\prime}-v u^{\prime}\right)\right]_{a}^{b}=0
$$$$
\left(P=e^{\int_{a}^{x} p d x}\right)
$$

On account of $(\gamma)$ the quantity

$$
-\left[P y y^{\prime}\right]_{a}^{b}
$$

is positive or zero for any function $y$ which satisfies (2). Under these assumptions the following theorem holds: $\dagger$

There exists an infinite series of normal parameter values $\lambda_{n}$ and corres.ponding solutions (normal functions) $y_{n}$ of the differential equation (1) and the boundary conditions (2). If $A$ changes sign in $(a, b)$, the values $\lambda_{n}$ include an infinite series of positive terms, $\lambda_{1} \leqq \lambda_{2} \leqq \lambda_{3} \leqq \lambda_{4} \leqq \ldots$, increasing without limit, and an infinite series of negative terms, $\lambda_{-1} \geqq \lambda_{-2} \geqq \lambda_{-3} \geqq \lambda_{-4} \geqq \ldots$, decreasing without limit. $\$$ The function $y_{n}$ satisfies the conditions $\S$

$$
\begin{aligned}
& \int_{a}^{b} P A y^{2} d x= \pm 1 \\
& \int_{a}^{b} P A y_{i} y d x=0 \quad[i= \pm 1, \pm 2, \cdots, \pm(n-1)]
\end{aligned}
$$

and gives to the expression

$$
J(y)=\int_{a}^{b}\left({y^{\prime 2}}^{2} B y^{2}\right) P d x-\left[y y^{\prime} P\right]_{a}^{b}
$$

its least possible value consistent with these conditions and with equations (2). This minimum value of $J$ is $\pm \lambda_{n}$.

On the basis of these properties the following theorem will be proved:

A function $f(x)$ which satisfies the boundary conditions (2), is continuous within $(a, b)$, and has a derivative which is continuous except at a finite number of points, may be expanded in a uniformly and absolutely converging series of the form

* See page 349 of the article cited.

† Ibid., page 356.

If $\boldsymbol{A}$ does not change sign, there are no normal parameter values of sign opposite to that of $A$. In case $B \equiv 0, \lambda_{1}$ or $\lambda_{-1}$ may be zero.

\& The upper or lower signs are to be taken according as $\lambda_{n}$ is positive or negative. 
where

$$
\begin{gathered}
c_{1} y_{1}+c_{2} y_{2}+c_{3} y_{3}+\cdots \\
+c_{-1} y_{-1}+c_{-2} y_{-2}+c_{-3} y_{-3}+\cdots
\end{gathered}
$$

$$
c_{ \pm n}= \pm \int_{a}^{b} f A y_{ \pm n} d x
$$

§1. A property of the constants $c_{i}$.

By consideration of the expression $J$ it will be proved that the series

are convergent.

$$
\sum_{i=1}^{\infty} c_{i}^{2} \lambda_{i}, \quad \sum_{i=-1}^{-\infty} c_{i}^{2} \lambda_{i}
$$

If $u$ and $v$ are two functions satisfying the boundary conditions, then

where

$$
J(u+v)=J(u)+J(v)+2 J_{1}(u, v),
$$

$$
J_{1}(u, v)=\int_{a}^{b}\left(u^{\prime} v^{\prime}+B u v\right) P d x-\left[u v^{\prime} P\right]_{a}^{b} .
$$

Then, since $J\left(y_{i}\right)=\lambda_{i}$ for positive subscripts,

(4) $J\left(f-\sum_{i=1}^{n} c_{i} y_{i}\right)=J(f)+\sum_{i=1}^{n} c_{i}^{2} \lambda_{i}-2 \sum_{i=1}^{n} c_{i} J_{1}\left(f, y_{i}\right)+2 \sum_{i, k=1}^{n} c_{i} c_{k} J_{1}\left(y_{i}, y_{k}\right)$.

Now the function $y_{i}$ satisfies the differential equation (1) for $\lambda=\lambda_{i}$, or on multiplying (1) by $P=e \int_{a}^{*}$ pdx

$$
\left(P y_{i}^{\prime}\right)^{\prime}+\left(\lambda_{i} A-B\right) P y_{i}=0 .
$$

Therefore,

Similarly,

$$
\begin{aligned}
J_{1}\left(f, y_{i}\right) & =\int_{a}^{b}\left(f^{\prime} y_{i}^{\prime}+B f y_{i}\right) P d x-\left[f y_{i}^{\prime} P\right]_{a}^{b} \\
& =\int_{a}^{b} f\left[B P y_{i}-\left(P y_{i}^{\prime}\right)^{\prime}\right] d x=\lambda_{i} \int_{a}^{b} P A f y_{i} d x=c_{i} \lambda_{i} .
\end{aligned}
$$

$$
J_{1}\left(y_{i}, y_{k}\right)=\lambda_{i} \int_{a}^{b} P A y_{i} y_{k} d x=0 .
$$

Since the left member of equation (4) is positive or zero, it follows that whatever be $n$,

$$
J(f) \geqq \sum_{i=1}^{n} c_{i}^{2} \lambda_{i} \cdot
$$

But the terms of the series are positive. Therefore the series

$$
\sum_{i=1}^{\infty} c_{i}^{2} \lambda_{i}
$$


is convergent. The convergence of the series with negative subscripts is proved in the same way by considering $J\left(f-\sum_{i=-1}^{-n} c_{i} y_{i}\right)$.

$\S 2$. Convergence of the series.

Consider the terms with positive subscripts, and write

$$
\phi_{n, m}=\sum_{i=n}^{n+m}\left|c_{i} y_{i}\right|
$$

If $n$ be taken sufficiently large, each function $y_{i}$ will have at least one zero $\alpha_{i}$ in the interval $(a, b)$, and

$$
\phi_{n, m}=\sum_{i=n}^{n+m}\left|c_{i} \int_{a_{i}}^{x} y_{i}^{\prime} d x\right| .
$$

Now $r^{2}+s^{2} \geqq 2 r s$ for any two numbers $r, s$, and therefore

$$
\phi_{n, m}^{2} \leqq 2 \sum_{i=n}^{n+m} c_{i}^{2}\left(\int_{a_{i}}^{x} y_{i}^{\prime} d x\right)^{2} .
$$

Furthermore, for all values of the parameter $\mu$, the expression

$$
\int_{a_{i}}^{x}\left(\mu+y_{i}^{\prime}\right)^{2} d x=\mu^{2}\left(x-\alpha_{i}\right)+2 \mu \int_{a_{i}}^{x} y_{i}^{\prime} d x+\int_{a_{i}}^{x} y_{i}^{\prime 2} d x
$$

has the sign of $x-\alpha_{i}$. The discriminant of this form is then not negative, that is,

But

$$
\left(\int_{\alpha_{i}}^{x} y_{i}^{\prime} d x\right)^{2} \leqq\left(x-\alpha_{i}\right) \int_{a_{i}}^{x} y_{i}^{\prime 2} d x \leqq(b-a) \int_{a}^{b} y_{i}^{\prime 2} d x
$$

and therefore

$$
\int_{a}^{b} y_{i}^{\prime 2} d x \leqq J\left(y_{i}\right)=\lambda_{i}
$$

Since the series

$$
\phi_{n, m}^{2} \leqq 2 \sum_{i=n}^{n+m} c_{i}^{2} \lambda_{i}
$$

$$
\sum_{i=1}^{\infty} c_{i}^{2} \lambda_{i}
$$

* This follows at once from one of the simplest of STURM's well-known results. It may be proved for the case here considered as follows: Let $u$ be a function having $\alpha$ and $\beta$ for consecutive zeros in an interval where $A>0$, and satisfying (1) for $\lambda=\bar{\lambda}$ inside $(\alpha, \beta)$. The existence of such a function is assured by the theorem stated in the introduction. From the differential equations for $u$ and $y_{i}$ it follows that

$$
\left[y_{i} u^{\prime} P\right]_{\alpha}^{\beta}=\left(\lambda_{i}-\bar{\lambda}\right) \int_{\alpha}^{\beta} P A u y_{i} d x .
$$

Determine the sign of $u$ to be positive in $(\alpha, \beta)$. Then $u^{\prime}(\beta)<0, u^{\prime}(\alpha)>0$, since $u$ cannot vanish together with its derivative. Now $A>0$ in $(\alpha, \beta)$, and therefore it follows from the above equation that $y_{i}$ must change sign in $(\alpha, \beta)$ if $\lambda_{i}>\bar{\lambda}$. 
is convergent, it follows that the series

$$
c_{1} y_{1}+c_{2} y_{2}+c_{3} y_{3}+\cdots
$$

converges uniformly and absolutely. The convergence of the series of terms with negative subscripts is proved in the same way.

\section{§3. The series represents the function.}

Since the series converges uniformly, the difference

$$
f-\sum_{i=-\infty}^{\infty} c_{i} y_{i}=g
$$

is a continuous function. On multiplying this equation by $P A y_{i}$ and integrating from $a$ to $b$, the equation

$$
\int_{a}^{b} P A y_{i} g d x=0
$$

results, whatever be $i$. This equation is sufficient to insure that $g$ is identically zero. It will be shown that on assumption of the contrary a function $y$ may be formed, which has a continuous derivative and satisfies the boundary conditions (2) and the equations

$$
\begin{aligned}
& \int_{a}^{b} P A y_{i} y d x=0 \quad(i= \pm 1, \pm 2, \pm 3, \cdots), \\
& \int_{a}^{b} P A y^{2} d x= \pm 1 .
\end{aligned}
$$

Suppose such a function exists. The value of the expression

$$
J=\int_{a}^{b} P\left(y^{\prime 2}+B y^{2}\right) d x-\left[y y^{\prime} P\right]_{a}^{b}
$$

is less than $\lambda_{n}$ and $-\lambda_{-n}$ if $n$ be taken sufficiently large, since these values increase without limit with $n$. Now $y$ satisfies all the conditions of the minimum problem of which either $y_{n}$ or else $y_{-n}$ is the solution, according as (7) holds with upper or lower sign. But it gives to $J$ a value less than the smallest value $\lambda_{n}$ or $-\lambda_{-n}$ consistent with the conditions. Such a function $y$ can therefore not exist.

It remains to show that if the difference $g$ is not identically zero, a function $y$ satisfying the boundary conditions (2) and the equations (6) and (7) can be formed. Let $\bar{\lambda}$ be a value which is not a normal parameter value. Then there exists a solution of the equation

$$
\left(P u^{\prime}\right)^{\prime}+(\bar{\lambda} A-B) P u=P A g
$$

under the boundary conditions (2). From this equation and 


$$
\left(P y_{i}^{\prime}\right)^{\prime}+\left(\lambda_{i} A-B\right) P y_{i}=0
$$

it follows, on account of (5), that

$$
\int_{a}^{b}\left\{y_{i}\left(P u^{\prime}\right)^{\prime}-u\left(P y_{i}^{\prime}\right)^{\prime}\right\} d x=\left(\lambda_{i}-\bar{\lambda}\right) \int_{a}^{b} P A y_{i} u d x .
$$

The left member vanishes after an integration by parts, on account of equation (3). Therefore, since $\bar{\lambda} \neq \lambda_{i}$, the equations (6) are satistied by the function $u$. On multiplying $u$ by a constant the equation (7) may be satisfied also, unless

$$
\int_{a}^{b} P A u^{2} d x=0 \text {. }
$$

Suppose, however, that this equation holds. Solve the equation

$$
\left(P v^{\prime}\right)^{\prime}+(\bar{\lambda} A-B) P v=P A u
$$

under the boundary conditions (2). This function satisfies (6), the proof being the same as before, except that $g$ is replaced by $u$. Hence the desired function $y$ may be formed by multiplying $v$ by a constant so that (7) will hold, unless

$$
\int_{a}^{b} P A v^{2} d x=0
$$

Suppose again that this is the case. Consider then the function

$$
w=u-v,
$$

which satisfies (2) and (6). Now

$\int_{a}^{b} P A w^{2} d x=-2 \int_{a}^{b} P A u v d x=-2 \int_{a}^{b}\left\{v\left(P v^{\prime}\right)^{\prime}-B P v^{2}\right\} d x=2 J(v)>0$.

The required function $y$ may, therefore, be formed in any case, unless $g$ is identically zero. It follows that the series represents the function.

At a later time $I$ hope to extend this method to more general cases.

Shemprired Scikntific School,

YALE UNIVTrRITY. 